\title{
Semi-empirical calculations of radiative decay rates in Mo II. A comparison between oscillator strength parametrization and core-polarization-corrected relativistic Hartree-Fock approaches
}

\author{
Safa Bouazza ${ }^{\mathrm{a}, *}$, Patrick Palmeri ${ }^{\mathrm{b}}$, Pascal Quinet ${ }^{\mathrm{b}, \mathrm{c}}$ \\ a Département de physique, Université de Reims-Champagne, UFR SEN, BP 1039, F-51687 Reims Cedex 2, France \\ ${ }^{\mathrm{b}}$ Physique Atomique et Astrophysique, Université de Mons- UMONS, B-7000 Mons, Belgium \\ ' $I P N A S$, Université de Liège, B-4000 Liège, Belgium
}

\section{A R T I C L E I N F O}

\section{Article history:}

Received 13 January 2017

Revised 23 March 2017

Accepted 17 May 2017

Available online 22 May 2017

\begin{abstract}
A B S T R A C T
We present a semi-empirical determination of Mo II radiative parameters in a wide wavelength range 1716-8789 ̊. Our fitting procedure to experimental oscillator strengths available in the literature permits us to provide reliable values for a large number of Mo II lines, predicting previously unmeasured oscillator strengths of lines involving $4 d^{4} 5 p$ and $4 d^{3} 5 s 5 p$ odd-parity configurations. The extracted transition radial integral values are compared with ab-initio calculations: on average they are 0.88 times the values obtained with the basic pseudo-relativistic Hartree Fock method and they agree well when core polarization effects are included. When making a survey of our present and previous studies and including also those given in the literature we observe as general trends a decreasing of transition radial integral values with filling nd shells of the same principal quantum numbers for $n \mathrm{~d}^{\mathrm{k}}(n+1) \mathrm{s} \rightarrow n \mathrm{~d}^{\mathrm{k}}(n+1) \mathrm{p}$ transitions.
\end{abstract}

(c) 2017 Elsevier Ltd. All rights reserved.

\section{Introduction}

Molybdenum is an element of the $4 \mathrm{~d}$-shell transition group. There are 33 known isotopes of molybdenum (Mo) ranging in atomic mass from 83 to 115 , as well as 4 metastable nuclear isomers. Seven isotopes occur naturally, with atomic masses of 92, 94, 95, 96, 97, 98, and 100. Historically, it is in 1926 that Meggers and Kiess initiated the level structure analysis of singly ionized molybdenum, establishing 27 levels [1]. Later on, Schauls and Sawyer [2] extended this first study. It is in 1958 that a big extension of this study occurred, performed by Kiess alone this time, giving furthermore 179 experimental Landé-factor values of levels [3]. This good compilation had constituted in its day a masterpiece of clarity and accuracy and showed the presence of strong intuition. More recently, after a break of a half century nearly a full fine structure (fs) study was published [4]: the spectrum of Mo II has been recorded with Fourier transform spectrometers in the wavelength interval $1500-7000 \AA$ and 110 new levels were reported. Furthermore an accurate theoretical analysis of 5 even-parity configurations and 2 odd-parity configurations was performed. In our recent analysis devoted mainly to Mo II hyperfine structure (hfs) we have dealt beforehand with fine structure level study [5] to ob-

\footnotetext{
* Corresponding author.

E-mail address: safa.bouazza@univ-reims.fr (S. Bouazza).
}

tain level eigenvectors, in order to work in intermediate coupling; we confirm the validity of the excellent huge work of $\mathrm{H}$. Nilsson and J. C. Pickering [4], since when we have recurred to the extracted level eigenvectors from this work in order to compute hfs data, the latter give a satisfactory agreement with new and accurate experimental hfs data of Rosner and Holt [6]. We have only to point out that the two levels of $\left({ }^{3} \mathrm{D}\right) 5 \mathrm{p} \mathrm{w} \mathrm{w}^{2} \mathrm{D}$, at $72,039.362 \mathrm{~cm}^{-1}$ and $72,829.914 \mathrm{~cm}^{-1}$ should have their total angular momentum $\mathrm{J}$ designations inverted $[3,5]$. In an extension of these Mo II electronic structure studies previously cited, relative experimental oscillator strengths of 58 Mo II lines were determined by Schnehage et al. [7] but their investigations covered only the region 2700$5700 \AA$; this limitation did not permit them to reach ranging in wavelength transitions involving the ground term levels. Historically, in the same year Hannaford and Lowe [8] measured lifetimes of 15 Mo II levels. Eighteen years later Sikström et al. [9] reported experimental branching fraction values, ranging in the wavelength from 1970 to $4370 \AA$ and radiative lifetime measurements for 10 levels. We have to mention also the complementary extensions on the same subject, done by Lundberg et al. [10] and Jiang et al. [11]. Recently a Spanish team has presented numerous new oscillator strength measurements, arising mainly from highly excited levels $[12,13]$. Laser-induced plasma generated from a fused glass sample was used to obtain radiative parameters via laser-induced breakdown spectroscopy. Theoretically, in [14] Quinet had calculated ra- 
diative lifetimes and oscillator strengths within the framework of the pseudo-relativistic Hartree Fock (HFR) method, including core polarisation effects. Nilsson and Pickering [4] present HFR oscillator strengths for all their classified lines. In turn in this study we propose to perform Mo II oscillator strength data recurring to semi-empirical oscillator strength parametrization (OSP) method and to compare our data with those given previously in the literature and to predict those which are missing with help of the pseudo-relativistic Hartree Fock (HFR) method, including core polarisation effects.

\section{Oscillator strength calculation}

We open this paragraph by evoking the inescapable relations we have made use in this work: the oscillator strength $f\left(\gamma \gamma^{\prime}\right)$ for the transition between two levels $\left.|\gamma|\right\rangle$ and $\left|\gamma^{\prime} J^{\prime}\right\rangle$ of an atom or molecule with statistical weights $g=(2 J+1)$ and $g^{\prime}=\left(2 J^{\prime}+1\right)$ respectively, is a dimensionless physical quantity, expressing the probability of absorption or emission in this transition between these two levels and related to the transition probability $W\left(\gamma \gamma^{\prime}\right)$ by [15]:

$W\left(\gamma \gamma^{\prime}\right)=\frac{2 \omega^{2} e^{2}}{m c^{3}} \mid f\left(\gamma \gamma^{\prime} \mid\right.$

where $m$ and $e$ are the electron mass and charge, $c$ is the velocity of light, $\gamma$ describes the initial quantum state, $\omega=\left[E(\gamma)-E\left(\gamma^{\prime}\right)\right] / \hbar$, $E(\gamma)$ is the energy of the initial state. The quantities with primes refer to the final state.

For the electric dipole transitions, the weighted oscillator strength $g f$ is related to the line strength $\boldsymbol{S}$ [15]:

$g f=8 \pi^{2} m c a_{0}^{2} \frac{\sigma}{3 h} S=303.76 \times 10^{-8} \sigma S$

where $\mathrm{a}_{0}$ is the Bohr radius, $\sigma=\left|E(\gamma)-E\left(\gamma^{\prime}\right)\right| / h c$ and $h$ is the Planck's constant.

The electric dipole line strength is defined by:

$S=\left|\left\langle\gamma J\left\|P^{1}\right\| \gamma^{\prime} J^{\prime}\right\rangle\right|^{2}$

The tensorial operator $\boldsymbol{P}^{\mathbf{1}}$ (in units of $\mathrm{ea}_{0}$ ) in the reduced matrix element stands for the electric dipole moment.

To obtain the $g f$ value, we need to calculate initially $\boldsymbol{S}$, or preferably its square root. For multiconfiguration system, the wavefunctions $|\gamma|>$ and $\mid \gamma^{\prime} J^{\prime}>$ are expanded in terms of a set of basis functions $\mid \phi S L J>$ and $\mid \phi^{\prime} S^{\prime} L^{\prime} J^{\prime}>$, respectively:

$|\gamma J\rangle=\sum_{i} c_{i}|\phi S L J\rangle \quad$ and $\quad\left|\gamma^{\prime} J^{\prime}\right\rangle=\sum_{j} c_{j}{ }^{\prime}\left|\phi^{\prime} S^{\prime} L^{\prime} J^{\prime}\right\rangle$

The square root of the line strength may be written in the following form:

$S_{\gamma \gamma^{\prime}}^{1 / 2}=\sum_{i} \sum_{j} c_{i} c_{j}{ }^{\prime}\left\langle\phi S L J\left\|P^{1}\right\| \phi^{\prime} S^{\prime} L^{\prime} J^{\prime}\right\rangle$

The appropriate computer program [16] calculates the angular part of the matrix element $<\phi S L J\left\|\mathrm{P}^{1}\right\| \phi^{\prime} S^{\prime} L^{\prime} J^{\prime}>$. From Eqs. (2) and (5), we can express the $g f$-values as a linear combination:

$$
\begin{aligned}
(g f)^{1 / 2}= & \sum_{n l, n^{\prime} l^{\prime}}\left(303.76 \times 10^{-8} \sigma\right)^{1 / 2} \\
& \times \sum_{i} \sum_{j} c_{i} c_{j}{ }_{j}\left\langle\phi S L J\left\|P^{1}\right\| \phi^{\prime} S^{\prime} L^{\prime} J^{\prime}\right\rangle
\end{aligned}
$$

where $\sigma$ is the wavenumber, given in $\mathrm{cm}^{-1}$, and the sum extends over all possible transitions ( $n \mathrm{~s} \leftrightarrow n^{\prime} \mathrm{p}, n \mathrm{~d} \leftrightarrow n^{\prime} \mathrm{p}, n \mathrm{~d} \leftrightarrow n^{\prime} \mathrm{f}$ ).

The weighted transition probability is [15]:

$g A=\left(2 J^{\prime}+1\right) A=\frac{64 \pi^{4} e^{2} a_{0}}{3 h} \sigma^{3} S=2.0261 \times 10^{-6} \sigma^{3} S$
Table 1

Mo II Transition radial integral values.

\begin{tabular}{llll}
\hline Transition & HFR $^{\mathrm{a}}$ & HFR+CPOL & OSP $^{\mathrm{c}}$ \\
\hline $4 \mathrm{~d}^{4} 5 \mathrm{~s}-4 \mathrm{~d}^{3} 5 \mathrm{~s} 5 \mathrm{p}$ & 1.8848 & 1.5224 & \\
$4 \mathrm{~d}^{4} 5 \mathrm{~s}-4 \mathrm{~d}^{4} 5 \mathrm{p}$ & 3.4247 & 2.9597 & $2.8558(0.0084)$ \\
$4 \mathrm{~d}^{5}-4 \mathrm{~d}^{4} 5 \mathrm{p}$ & 2.0962 & 1.7665 & $1.725(0.010)$ \\
$4 \mathrm{~d}^{3} 5 \mathrm{~s}^{2}-4 \mathrm{~d}^{3} 5 \mathrm{~s} 5 \mathrm{p}$ & 3.2856 & 2.7753 & \\
\hline a Relativistic Hartree Fock method & \\
b Relativistic Hartree Fock method including core-polarization \\
c Oscillator strength parametrization method
\end{tabular}

where $\sigma$ is given, as previously, in $\mathrm{cm}^{-1}$ and $S$ in atomic units of $\mathrm{e}^{2} \mathrm{a}{ }_{0}^{2}$. Substitution of Eq. (1) into (7) leads to:

$\left(2 J^{\prime}+1\right) A=0.66702 \sigma^{2} g f$

In the present study, our aim is to evaluate at first line strengths using Eq. (5). As in previous work devoted to oscillator strength determination (see for instance [16]), the angular coefficients of the transition matrix, obtained in pure $S L$ coupling with help of Racah algebra is transformed into the actual intermediate coupling recurring to our level eigenvector amplitudes previously derived. Moreover the transition integrals

$\int_{0}^{\infty} P_{n l}(r) r P_{n^{\prime} l^{\prime}}(r) d r$

are treated as free parameters in the least squares fit to experimental gf values. Up to now only $4 d^{5}-4 d^{4} 5 p$ and $4 d^{4} 5 s-4 d^{4} 5 p$ Mo II transition oscillator strengths have been measured. We have initially selected the strongest lines, not blended, whose experimental gf values were published very recently [12,13], with the best accuracy and particularly those representing transitions between levels with a limited number of leading components as shown in [4]; then, for these $4 d^{4} 5 s-4 d^{4} 5 p$ and $4 d^{5}-4 d^{4} 5 p$ transitions we have extracted semi-empirically, with very good accuracy, the radial integral values, reported in Table 1 . In the same table, we also give the results computed by means of the pseudo-relativistic Hartree Fock (HFR) method using the basic Cowan code [17] and including core-polarization (HFR+CPOL), as described in $[18,19]$. As a reminder, in the latter approach, the radial dipole integrals given in Eq. (9) are replaced by

$$
\begin{gathered}
\int_{0}^{\infty} P_{n l}(r) r\left[1-\frac{\alpha_{d}}{\left(r^{2}+r_{c}^{2}\right)^{3 / 2}}\right] P_{n^{\prime} l^{\prime}}(r) d r \\
-\frac{\alpha_{d}}{r_{c}^{3}} \int_{0}^{r_{c}} P_{n l}(r) r P_{n^{\prime} l^{\prime}}(r) d r
\end{gathered}
$$

where $a_{d}$ is the dipole polarizability of the ionic core, for which numerical values can be found in the literature (see e.g. [20]), and $r_{c}$ is the cut-off radius that is arbitrarily chosen as a measure of the size of the ionic core. In practice, this parameter is usually chosen to be equal to the HFR mean value $<r>$ for the outermost ionic core orbital.

When looking at Table 1, one can observe that ab initio HFR radial dipole integrals are about $20 \%$ larger than the values extracted from our oscillator strength parametrization (OSP) approach while the difference is reduced to a few percent when considering the HFR + CPOL results obtained using a Mo IV ionic core, as reported in $[10,11]$. In the same Table, for further analysis, we have also added HFR and HFR+CPOL radial integral values for transitions involving the $4 d^{3} 5 s 5 p$ configuration.

In Table 2, we have gathered the radial integral values of $n \mathrm{~d}^{\mathrm{k}}(n+1) \mathrm{s}->n \mathrm{~d}^{\mathrm{k}}(n+1) \mathrm{p}$ transitions, obtained semi-empirically in our previous works, using the same code, for singly ionized atoms, such as V II [21], Zr II [22], Nb II [23], Rh II [24], Hf II [25] to which we have added those given by Ruczkowski et al. for Sc II [16] and Ti II [26]. It is easy to observe that these transition radial 
Table 2

$\mathrm{ns} \rightarrow \mathrm{np}$ transition radial integral values of some singly ionized atoms.

\begin{tabular}{|c|c|c|c|c|c|c|c|}
\hline ion & Z & Transition & $\mathrm{HFR}^{\mathrm{a}}$ & $\mathrm{HFR}+\mathrm{CPOL}(\mathrm{III})^{\mathrm{b}}$ & $\mathrm{HFR}+\mathrm{CPOL}(\mathrm{IV})^{\mathrm{c}}$ & OSP $^{d}$ & OSP/HFR \\
\hline Sc II & 20 & $3 \mathrm{~d} 4 \mathrm{~s} 3 \mathrm{~d} 4 \mathrm{p}$ & 3.6199 & 3.2827 & 3.4201 & $3.413(0.018)[26]$ & 0.94 \\
\hline Ti II & 22 & $3 d^{2} 4 s \quad 3 d^{2} 4 p$ & 3.4511 & 3.0921 & 3.2530 & $3.078(0.015)[21]$ & 0.89 \\
\hline V II & 23 & $3 d^{3} 4 s \quad 3 d^{3} 4 p$ & 3.3085 & 2.9334 & 3.1035 & $3.0346(0.0094)[27]$ & 0.92 \\
\hline Zr II & 40 & $4 d^{2} 5 s \quad 4 d^{2} 5 p$ & 3.7111 & 2.9731 & 3.3361 & $3.15(0.02)[22]$ & 0.85 \\
\hline $\mathrm{Nb}$ II & 41 & $4 d^{3} 5 s 4 d^{3} 5 p$ & 3.5556 & 2.7842 & 3.1144 & $3.0605(0.0059)[23]$ & 0.86 \\
\hline Mo II & 42 & $4 d^{4} 5 s \quad 4 d^{4} 5 p$ & 3.4247 & 2.6478 & 2.9597 & $2.8558(0.0084)$ & 0.83 \\
\hline Rh II & 45 & $4 d^{7} 5 s \quad 4 d^{7} 5 p$ & 3.1238 & 2.3680 & 2.6374 & $2.7426(0.0007)[24]$ & 0.88 \\
\hline Hf II & 72 & $5 d^{2} 6 s 5 d^{2} 6 p$ & 3.4833 & 2.6500 & 3.0284 & $2.984 *(0.013)[25]$ & 0.86 \\
\hline
\end{tabular}

a Relativistic Hartree Fock method.

b Relativistic Hartree Fock method including core-polarization with a $2+$ ionic core.

c Relativistic Hartree Fock method including core-polarization with a $3+$ ionic core.

d Oscillator strength parametrization method.

* There is a typo in paper [25] from where we have taken this value.

integral values decrease with the filling of $n d$-shells for the same principal quantum number; this behaviour is different for instance from established general trends in the hyperfine structure analyses: increasing (contrary to the transition radial integral which is rather decreasing) of the most influential s-monoelectronic hfs parameter divided by $g_{I}=\mu_{I} / I, \mathrm{a}_{n s}^{10} / g_{I}$ versus atomic number $\mathrm{Z}$ [27]. These remarks may serve, with resorting to any calculations, as hints at the starting of oscillator strength fitting procedure since we can use the deduced interval of our new investigated transition radial integral values with the help of those known for other ions and then we can conclude if our obtained data in the first stage are encouraging or not to carry on with our fitting procedure. For instance we propose intuitively $<n \mathrm{~d}^{\mathrm{k}}(n+1) \mathrm{s}\left|r^{1}\right| n \mathrm{~d}^{\mathrm{k}}(n+1) \mathrm{p}>=3.00$ (0.02), $2.82(0.02)$ and $2.78(0.02)$ respectively for $3 d^{4} 4 s-3 d^{4} 4 p$ in Cr II, $4 d^{5} 5 s-4 d^{5} 5 p$ in Tc II and $4 d^{6} 5 s-4 d^{6} 5 p$ in Ru II when having recourse to Table 2 (the value between parentheses shows the uncertainty).

In the latter table, we also give the radial dipole integrals calculated using the pseudo-relativistic Hartree Fock method without and with core-polarization corrections. It is interesting to note that the values deduced from our oscillator strength parametrization approach are on average $12 \%$ smaller than ab initio HFR results. It is also worthwhile to underline that the OSP data are generally very close to the HFR + CPOL values provided that an ionic core of the type of triply ionized species (IV) is considered in the latter approach. This can be rather easily explained by the fact that core-polarization effects are partially included in OSP calculations when considering configurations of the type $n \mathrm{~d}^{\mathrm{k}-1}(n+1) \mathrm{s}^{2}$ and $n \mathrm{~d}^{\mathrm{k}-1}(n+1) \mathrm{s}(n+1) \mathrm{p}$, which correspond, for singly charged atoms, to a $3+$ ionic core surrounded by 2 electrons.

\section{Radiative data}

The oscillator strengths obtained in our work with the semiempirical oscillator strength parametrization (OSP) method are compared with the available experimental values $[9,12,13]$ in Table 3 for Mo II spectral lines in the region 2000-4000 $\mathrm{A}$ They are also compared with the values calculated using the HFR+CPOL approach. As the physical model considered in the latter method was exactly the same as the one described in our recent study of singly ionized molybdenum [10,11], the details will not be repeated here. Let us just remind that, in these works, the configurations $4 d^{5}, 4 d^{4} 5 s, 4 d^{4} 6 s, 4 d^{4} 5 d, 4 d^{3} 5 s^{2}, 4 d^{3} 5 p^{2}, 4 d^{3} 5 d^{2}, 4 d^{3} 5 s 5 d$ (even parity) and $4 d^{4} 5 p, 4 d^{4} 6 p, 4 d^{4} 4 f, 4 d^{4} 5 f, 4 d^{3} 5 s 5 p, 4 d^{3} 5 p 5 d$ (odd parity) were included in the calculations with the dipole polarizability $\alpha_{\mathrm{d}}=5.67 \mathrm{a}_{0}{ }^{3}$ [20] and the cut off radius $\mathrm{r}_{\mathrm{c}}=1.73 \mathrm{a}_{0}$ as core-polarization parameters. This model was then combined with a semi-empirical adjustment of the radial parameters using the experimental energy levels published by Nilsson and Pickering [4], allowing us to optimize the average energies, the electrostatic interaction integrals and the spin-orbit parameters of $4 d^{5}, 4 d^{4} 5 s, 4 d^{4} 6 s$,

Table 3

Comparison between calculated and experimental oscillator strengths in Mo II.

\begin{tabular}{|c|c|c|c|c|c|c|c|c|}
\hline \multirow[t]{2}{*}{$\lambda(\AA)^{\mathrm{a}}$} & \multicolumn{2}{|c|}{ Lower level $^{\mathrm{b}}$} & \multicolumn{2}{|c|}{ Upper level $^{\mathrm{b}}$} & \multicolumn{4}{|l|}{$\log g f$} \\
\hline & $\mathrm{E}\left(\mathrm{cm}^{-1}\right)$ & $\mathrm{J}$ & $\mathrm{E}\left(\mathrm{cm}^{-1}\right)$ & $\mathrm{J}$ & $\mathrm{EXP}^{\mathrm{c}}$ & $\Delta \mathrm{gf}_{(\exp )}$ & OSPd $^{\mathrm{d}}$ & $\mathrm{HFR}+\mathrm{CPOL}^{\mathrm{d}}$ \\
\hline 2079.985 & 15,331 & $7 / 2$ & 63,393 & $5 / 2$ & -0.78 & 0.034 & -0.91 & -0.85 \\
\hline 2080.473 & 15,447 & $11 / 2$ & 63,498 & $9 / 2$ & -0.26 & 0.017 & -0.16 & -0.45 \\
\hline 2081.680 & 0 & $5 / 2$ & 48,023 & $3 / 2$ & -0.86 & 0.059 & -0.85 & -1.15 \\
\hline 2111.248 & 15,691 & $5 / 2$ & 63,042 & $5 / 2$ & -1.04 & 0.045 & -0.95 & -2.06 \\
\hline 2113.819 & 15,199 & $5 / 2$ & 62,492 & $7 / 2$ & -1.15 & 0.011 & -1.23 & -1.75 \\
\hline 2119.710 & 15,331 & $7 / 2$ & 62,492 & $7 / 2$ & -0.76 & 0.043 & -0.89 & -0.94 \\
\hline 2122.698 & 15,331 & $7 / 2$ & 62,426 & $5 / 2$ & -1.07 & 0.120 & -1.00 & -1.74 \\
\hline 2170.614 & 15,691 & $5 / 2$ & 61,747 & $3 / 2$ & -1.65 & 0.024 & -1.57 & -1.75 \\
\hline 2187.622 & 17,344 & $5 / 2$ & 63,042 & $5 / 2$ & -1.07 & 0.012 & -1.21 & -1.57 \\
\hline 2341.592 & 15,199 & $5 / 2$ & 57,892 & $7 / 2$ & -0.61 & 0.110 & -0.81 & -0.58 \\
\hline 2467.337 & 22,980 & $11 / 2$ & 63,498 & $9 / 2$ & -0.66 & 0.150 & -0.84 & -1.00 \\
\hline 2515.080 & 22,980 & $11 / 2$ & 62,729 & $11 / 2$ & -0.45 & 0.005 & -0.52 & -0.86 \\
\hline 2538.457 & 13,461 & $9 / 2$ & 52,843 & $9 / 2$ & -0.38 & 0.19 & -0.31 & -0.37 \\
\hline 2542.672 & 12,901 & $7 / 2$ & 52,218 & $7 / 2$ & -0.58 & 0.082 & -0.79 & -0.84 \\
\hline 2551.021 & 23,854 & $7 / 2$ & 63,042 & $5 / 2$ & -1.81 & 0.012 & -2.00 & -1.99 \\
\hline 2588.779 & 23,935 & $5 / 2$ & 62,551 & $3 / 2$ & -0.86 & 0.028 & -0.89 & -0.59 \\
\hline 2596.420 & 24,509 & $7 / 2$ & 63,013 & $9 / 2$ & -1.36 & 0.004 & -1.57 & -1.51 \\
\hline 2602.800 & 11,784 & $1 / 2$ & 50,192 & $3 / 2$ & -0.60 & 0.170 & -0.58 & -0.69 \\
\hline 2618.465 & 24,372 & $1 / 2$ & 62,551 & $3 / 2$ & -1.14 & 0.105 & -1.10 & -3.30 \\
\hline
\end{tabular}

(continued on next page) 
Table 3 (continued)

\begin{tabular}{|c|c|c|c|c|c|c|c|c|}
\hline \multirow[t]{2}{*}{$\lambda(\AA)^{\mathrm{a}}$} & \multicolumn{2}{|c|}{ Lower level $^{\mathrm{b}}$} & \multicolumn{2}{|c|}{ Upper level $^{\mathrm{b}}$} & \multicolumn{4}{|l|}{$\log g f$} \\
\hline & $\mathrm{E}\left(\mathrm{cm}^{-1}\right)$ & $\mathrm{J}$ & $\mathrm{E}\left(\mathrm{cm}^{-1}\right)$ & $\mathrm{J}$ & $\mathrm{EXP}^{c}$ & $\Delta \mathrm{gf}_{(\exp )}$ & OSP $^{d}$ & $\mathrm{HFR}+\mathrm{CPOL}^{\mathrm{d}}$ \\
\hline 2619.344 & 11,784 & $1 / 2$ & 49,950 & $1 / 2$ & -1.03 & 0.083 & -0.96 & -1.08 \\
\hline 2620.050 & 25,342 & $7 / 2$ & 63,498 & $9 / 2$ & -0.88 & 0.010 & -1.00 & -1.03 \\
\hline 2638.761 & 12,418 & $5 / 2$ & 50,303 & $7 / 2$ & -0.06 & 0.050 & 0.03 & -0.07 \\
\hline 2646.486 & 12,418 & $5 / 2$ & 50,192 & $3 / 2$ & -0.29 & 0.260 & -0.27 & -0.38 \\
\hline 2653.796 & 25,342 & $7 / 2$ & 63,013 & $9 / 2$ & -0.76 & 0.010 & -0.67 & -0.87 \\
\hline 2672.843 & 12,901 & $7 / 2$ & 50,303 & $7 / 2$ & 0.05 & 0.029 & 0.06 & -0.06 \\
\hline 2673.270 & 15,447 & $11 / 2$ & 52,843 & $9 / 2$ & -0.12 & 0.054 & -0.21 & -0.29 \\
\hline 2683.232 & 11,784 & $1 / 2$ & 49,041 & $3 / 2$ & -0.23 & 0.045 & -0.22 & -0.28 \\
\hline 2701.415 & 12,034 & $3 / 2$ & 49,041 & $3 / 2$ & -0.34 & 0.058 & -0.30 & -0.33 \\
\hline 2701.868 & 26,041 & $7 / 2$ & 63,042 & $5 / 2$ & -0.41 & 0.010 & -0.39 & -0.30 \\
\hline 2717.347 & 15,428 & $9 / 2$ & 52,218 & $7 / 2$ & -0.41 & 0.009 & -0.30 & -0.37 \\
\hline 2729.683 & 12,418 & $5 / 2$ & 49,041 & $3 / 2$ & -0.92 & 0.024 & -1.03 & -0.95 \\
\hline 2730.931 & 26,406 & $7 / 2$ & 63,013 & $9 / 2$ & -1.06 & 0.023 & -1.09 & -0.90 \\
\hline 2732.880 & 12,901 & $7 / 2$ & 49,481 & $7 / 2$ & -0.47 & 0.027 & -0.56 & -0.56 \\
\hline 2747.619 & 26,041 & $7 / 2$ & 62,426 & $5 / 2$ & -0.41 & 0.029 & -0.39 & -1.29 \\
\hline 2763.616 & 15,199 & $5 / 2$ & 51,373 & $3 / 2$ & -0.56 & 0.039 & -0.55 & -0.61 \\
\hline 2775.402 & 13,461 & $9 / 2$ & 49,481 & $7 / 2$ & 0.27 & 0.013 & 0.32 & 0.29 \\
\hline 2776.675 & 26,488 & $9 / 2$ & 62,492 & $7 / 2$ & -0.58 & 0.013 & -0.54 & -0.69 \\
\hline 2788.825 & 23,833 & $9 / 2$ & 59,680 & $9 / 2$ & -1.33 & 0.010 & -1.23 & -1.92 \\
\hline 2807.753 & 12,418 & $5 / 2$ & 48,023 & $3 / 2$ & -0.38 & 0.057 & -0.33 & -0.43 \\
\hline 2816.157 & 13,461 & $9 / 2$ & 48,960 & $11 / 2$ & 0.51 & 0.024 & 0.55 & 0.54 \\
\hline 2842.148 & 12,034 & $3 / 2$ & 47,209 & $1 / 2$ & -0.87 & 0.021 & -0.85 & -0.92 \\
\hline 2848.233 & 12,901 & $7 / 2$ & 48,000 & $9 / 2$ & 0.30 & 0.027 & 0.33 & 0.32 \\
\hline 2855.917 & 24,836 & $5 / 2$ & 59,841 & $3 / 2$ & -1.11 & 0.010 & -1.31 & -2.53 \\
\hline 2866.540 & 15,428 & $9 / 2$ & 50,303 & $7 / 2$ & -1.42 & 0.037 & -1.61 & -1.57 \\
\hline 2871.512 & 12,418 & $5 / 2$ & 47,232 & $7 / 2$ & 0.10 & 0.010 & 0.13 & 0.11 \\
\hline 2890.993 & 12,034 & $3 / 2$ & 46,614 & $5 / 2$ & -0.08 & 0.010 & -0.12 & -0.14 \\
\hline 2894.451 & 13,461 & $9 / 2$ & 48,000 & $9 / 2$ & -0.10 & 0.009 & -0.15 & -0.17 \\
\hline 2909.116 & 11,784 & $1 / 2$ & 46,148 & $3 / 2$ & -0.50 & 0.120 & -0.47 & -0.49 \\
\hline 2911.917 & 12,901 & $7 / 2$ & 47,232 & $7 / 2$ & -0.10 & 0.009 & -0.06 & -0.07 \\
\hline 2913.806 & 28,989 & $7 / 2$ & 63,299 & $7 / 2$ & -0.37 & 0.016 & -0.51 & -0.38 \\
\hline 2923.391 & 12,418 & $5 / 2$ & 46,614 & $5 / 2$ & -0.12 & 0.010 & -0.08 & -0.10 \\
\hline 2926.153 & 28,877 & $5 / 2$ & 63,042 & $5 / 2$ & -0.55 & 0.019 & -0.54 & -0.75 \\
\hline 2930.502 & 12,034 & $3 / 2$ & 46,148 & $3 / 2$ & -0.18 & 0.031 & -0.19 & -0.21 \\
\hline 2934.297 & 11,784 & $1 / 2$ & 45,853 & $1 / 2$ & -0.40 & 0.120 & -0.36 & -0.37 \\
\hline 2935.776 & 28,989 & $7 / 2$ & 63,042 & $5 / 2$ & -1.00 & 0.006 & -0.97 & -1.25 \\
\hline 2938.300 & 28,989 & $7 / 2$ & 63,013 & $9 / 2$ & -0.38 & 0.008 & -0.39 & -0.26 \\
\hline 2956.056 & 12,034 & $3 / 2$ & 45,853 & $1 / 2$ & -0.77 & 0.007 & -0.91 & -0.94 \\
\hline 2960.241 & 13,461 & $9 / 2$ & 47,232 & $7 / 2$ & -1.30 & 0.003 & -1.26 & -1.28 \\
\hline 2963.797 & 12,418 & $5 / 2$ & 46,148 & $3 / 2$ & -0.90 & 0.011 & -0.87 & -0.88 \\
\hline 2965.280 & 12,901 & $7 / 2$ & 46,614 & $5 / 2$ & -0.96 & 0.011 & -0.97 & -0.98 \\
\hline 2975.404 & 29,699 & $5 / 2$ & 63,299 & $7 / 2$ & -0.12 & 0.041 & -0.15 & -0.05 \\
\hline 3030.070 & 30,020 & $7 / 2$ & 63,013 & $9 / 2$ & -1.32 & 0.009 & -1.46 & -1.66 \\
\hline 3052.322 & 26,740 & $11 / 2$ & 59,492 & $13 / 2$ & -0.65 & 0.022 & -0.69 & -0.82 \\
\hline 3054.930 & 29,022 & $5 / 2$ & 61,747 & $3 / 2$ & -1.06 & 0.009 & -1.15 & -0.82 \\
\hline 3078.633 & 30,020 & $7 / 2$ & 62,492 & $7 / 2$ & -1.17 & 0.058 & -1.18 & -1.19 \\
\hline 3087.620 & 27,114 & $13 / 2$ & 59,492 & $13 / 2$ & 0.36 & 0.028 & 0.49 & 0.43 \\
\hline 3097.687 & 26,488 & $9 / 2$ & 58,761 & $11 / 2$ & -0.73 & 0.009 & -0.76 & -0.81 \\
\hline 3111.636 & 26,069 & $9 / 2$ & 58,197 & $9 / 2$ & -0.92 & 0.018 & -1.03 & -1.13 \\
\hline 3136.466 & 23,833 & $9 / 2$ & 55,707 & $7 / 2$ & -0.55 & 0.065 & -0.53 & -0.46 \\
\hline 3144.625 & 26,406 & $7 / 2$ & 58,197 & $9 / 2$ & -1.03 & 0.038 & -1.13 & -1.29 \\
\hline 3158.933 & 27,114 & $13 / 2$ & 58,761 & $11 / 2$ & -1.05 & 0.006 & -0.95 & -1.03 \\
\hline 3175.051 & 26,406 & $7 / 2$ & 57,892 & $7 / 2$ & -0.27 & 0.022 & -0.16 & -0.32 \\
\hline 3178.004 & 26,740 & $11 / 2$ & 58,197 & $9 / 2$ & -0.98 & 0.006 & -0.84 & -0.94 \\
\hline 3183.405 & 26,488 & $9 / 2$ & 57,892 & $7 / 2$ & -0.94 & 0.022 & -0.89 & -1.04 \\
\hline 3258.677 & 17,344 & $5 / 2$ & 48,023 & $3 / 2$ & -1.47 & 0.011 & -1.48 & -1.40 \\
\hline 3267.633 & 25,113 & $5 / 2$ & 55,707 & $7 / 2$ & -1.00 & 0.040 & -1.11 & -0.81 \\
\hline 3292.313 & 25,342 & $7 / 2$ & 55,707 & $7 / 2$ & 0.05 & 0.006 & -0.01 & 0.18 \\
\hline 3347.266 & 24,372 & $1 / 2$ & 54,239 & $1 / 2$ & -0.81 & 0.012 & -1.02 & -0.70 \\
\hline 3367.959 & 33,046 & $9 / 2$ & 62,729 & $11 / 2$ & -0.11 & 0.011 & -0.02 & 0.14 \\
\hline 3374.833 & 32,124 & $1 / 2$ & 61,747 & $3 / 2$ & -1.26 & 0.030 & -1.34 & -1.40 \\
\hline 3379.755 & 24,660 & $3 / 2$ & 54,239 & $1 / 2$ & -0.81 & 0.002 & -0.91 & -0.68 \\
\hline 3435.376 & 30,392 & $11 / 2$ & 59,492 & $13 / 2$ & -0.34 & 0.041 & -0.31 & -0.44 \\
\hline 3445.500 & 28,877 & $5 / 2$ & 57,892 & $7 / 2$ & -0.41 & 0.013 & -0.51 & -0.63 \\
\hline 3545.989 & 29,699 & $5 / 2$ & 57,892 & $7 / 2$ & -1.03 & 0.065 & -0.96 & -1.00 \\
\hline 3547.942 & 30,020 & $7 / 2$ & 58,197 & $9 / 2$ & -1.08 & 0.040 & -0.95 & -1.00 \\
\hline 3618.359 & 35,100 & $13 / 2$ & 62,729 & $11 / 2$ & -1.34 & 0.021 & -1.33 & -1.39 \\
\hline 3658.959 & 35,406 & $11 / 2$ & 62,729 & $11 / 2$ & -0.76 & 0.017 & -0.77 & -0.69 \\
\hline 3857.196 & 24,660 & $3 / 2$ & 50,578 & $5 / 2$ & -0.99 & 0.052 & -0.97 & -1.41 \\
\hline
\end{tabular}

a The wavelengths, given in vacuum (air) below (above) $2000 \AA$ are deduced from the experimental energy level values.

b Experimental energy levels taken from [4].

c Experimental values deduced from $[9,12,13]$.

d Values obtained in the present work using the oscillator strength parametrization (OSP) and corepolarization-corrected relativistic Hartree Fock (HFR+CPOL) methods. 
$4 d^{4} 5 d$ and $4 d^{4} 5 p, 4 d^{3} 5 s 5 p$ configurations in the even and odd parities, respectively.

As seen from Table 3, the OSP results are in very good agreement with the experimental oscillator strengths, the average ratio $g f_{\text {OSP }} / g f_{\text {EXP }}$ being equal to $0.98 \pm 0.20$, where the uncertainty corresponds to the standard deviation to the mean. A similar ratio, i.e. $0.94 \pm 0.30$, is found when considering the comparison between HFR+CPOL and experimental results, provided that the computed gf-values corresponding to transitions affected by strong cancellation effects are rejected from the mean, namely those appearing at $\lambda=2111.248,2113.819,2122.698,2187.622,2515.080,2618.465$, $2747.619,2855.917$, and $3857.196 \AA$. For these lines, it was indeed observed that the cancellation factor, $C F$, as defined by Cowan [17] took very small values $(C F<0.05)$ in our $\mathrm{HFR}+\mathrm{CPOL}$ calculations, indicating that the corresponding oscillator strengths could not be reliable.

Finally, in a Supplementary file, we give the oscillator strengths and transition probabilities computed in the present work using the HFR+CPOL method for about 1800 Mo II spectral lines involving available experimental energy levels with calculated log-gf values greater than -2 . This table covers a wide range of wavelengths, from 1750 to $8700 \AA$ and includes many new radiative parameters, not only for $4 d^{5}-4 d^{4} 5 p$ and $4 d^{4} 5 s-4 d^{4} 5 p$ transitions, but also for $4 d^{4} 5 s-4 d^{3} 5 s 5 p$ and $4 d^{3} 5 s^{2}-4 d^{3} 5 s 5 p$ transitions.

\section{Conclusion}

A new investigation of radiative decay rates for transitions in Mo II using the semi-empirical oscillator strength parametrization approach and the relativistic Hartree Fock method including core-polarization effects is reported in the present work. It is shown that both methods give a similar good agreement when compared to available experimental gf-values. It is also observed that the $4 \mathrm{~d}^{4} 5 \mathrm{~s} 4 \mathrm{~d}^{4} 5 \mathrm{p}$ radial dipole integral deduced from our oscillator strength parametrization approach has a similar value to the one obtained with the HFR+CPOL method. The trend is confirmed when we compare the OSP and HFR+CPOL $n \mathrm{~d}^{\mathrm{k}}(n+1) \mathrm{s} \rightarrow n \mathrm{~d}^{\mathrm{k}}(n+1) \mathrm{p}$ transition radial integrals in the case of other singly ionized atoms, such as Sc II, Ti II, V II, Zr II, Nb II, Rh II and Hf II.

\section{Acknowledgments}

PP and PQ are respectively Research Associate and Research Director of the Belgian Fund for Scientific Research F.R.S.-FNRS. Financial support from this organization is deeply acknowledged.

SB thanks deeply Dr J. Ruczkowski and Dr M. Elantkowska for providing him with their program package for oscillator strength parametrization.

\section{Supplementary materials}

Supplementary material associated with this article can be found, in the online version, at doi:10.1016/j.jqsrt.2017.05.019.

\section{References}

[1] Meggers WF, Kiess CC. Spectral structures for elements on the second long period. J Opt Soc Am 1926;12:417.

[2] Schauls R, Sawyer RA. New lines and terms in the arc and first spark spectra of molybdenum: Mo I and Mo II. Phys Rev 1940;58:784.

[3] C C Kiess J. Description and analysis of the second spectrum of molybdenum, Mo II. Res Nat Bur Std 1958;60 375 RP2856.

[4] Nilsson H, Pickering JC. Extended Term Analysis of Mo II. Phys Scr 2003;67:223

[5] Bouazza S. Full hyperfine structure analysis of singly ionized molybdenum. J Quant Spectrosc Radiat Transfer 2017;189:1.

[6] Rosner SD, Holt RA. Hyperfine structure and isotope shifts in Mo II. Can J Phys (doi:10.1139/CJP-2016-0192).

[7] Schnehage SE, Danzmann K, Künnemeyer R, Kock M. Experimental Mo II oscillator strengths. J Spectrosc Radiat Transfer 1983;39:507.

[8] Hannaford P, Lowe RM. Radiative lifetimes in Mo II. J Phys B 1983;16:4539.

[9] Sikström CM, Pihlemark H, Nilsson H, Litzén U, Johansson S, Li ZS, et al. Experimental Mo II oscillator strengths. J Phys B 2001;34:477.

[10] Lundberg $H$, Engström L, Hartman $H$, Nilsson $H$, Palmeri $P$, Quinet $P$, et al. Lifetime measurements and transition probabilities in Mo II. J Phys B 2010;43:085004.

[11] Jiang LY, Wang Q, Feng YY, Quinet P, Biémont E, Li SB, et al. Experimental and theoretical radiative properties of odd-parity highly excited levels in Mo II. Eur Phys J D 2012;66:176.

[12] Mayo-Garcia R, Aragon C, Aguilera JA, Ortiz M. Measured oscillator strengths in singly ionized molybdenum. J Phys B 2015:48:215002.

[13] Aragon C, Aguilera JA, Ortiz M, Mayo-Garcia R. Experimental oscillator strengths of highly excited levels of Mo II. J Phys B 2016;49:105003.

[14] Quinet P. Investigation of Configuration interaction and core polarisation effects in Mo II. J Phys B 2002;35:19.

[15] Sobelman I. Atomic spectra and radiative transitions. Berlin: Springer-Verlag; 1978.

[16] Ruczkowski J, Elantkowska M, Dembczynski J. An alternative method for determination of oscillator strengths. The example of Sc II. JQuant Spectrosc Radiat Transfer 2014;145:20.

[17] Cowan RD. The theory of atomic structure and spectra. Berkeley: California University Press; 1981.

[18] Quinet P, Palmeri P, Biémont E, McCurdy MM, Rieger G, Pinnington EH, et al. Experimental and theoretical lifetimes, branching fractions and oscillator strengths in Lu I. Mon Not R Astron Soc 1999;307:934.

[19] Quinet P, Palmeri P, Biémont E, Li ZS, Zhang ZG, Svanberg S. Radiative lifetime measurements and transition probability calculations in lanthanide ions. J Alloys Comp 2002;344:255.

[20] Fraga S, Karwowski J, Saxena KMS. Handbook of atomic data. Amsterdam: Elsevier Scientific Publishing Company; 1976.

[21] Bouazza S, Ruczkowski J, Elantkowska M, Dembczynski J. Hyperfine structure, lifetimes and oscillator strengths of V II. J Quant Spectrosc Radiat Transfer 2015;166:55.

[22] Quinet P, Bouazza S, Palmeri P. A comparative study between semi-empirical oscillator strength parametrization and relativistic Hartree-Fock methods for computing the radiative parameters in Zr II. J Quant Spectrosc Radiat Transfer 2015; 164:193.

[23] Ruczkowski J, Bouazza S, Elantkowska M, Dembczynski J. Semi-empirical analysis of oscillator strengths for Nb II. J Quant Spectrosc Radiat Transfer 2015;155:1.

[24] Bouazza S. Oscillator strengths and branching fractions of $4 \mathrm{~d} 75 \mathrm{p}-4 \mathrm{~d} 75 \mathrm{~s}$ Rh II transitions. J Quant Spectrosc Radiat Transfer 2017;187:280.

[25] Bouazza S, Quinet P. Palmeri P. Semi-empirical studies of atomic transition probabilities, oscillator strengths and radiative lifetimes in Hf II. J Quant Spectrosc Radiat Transfer 2015;163:39.

[26] Ruczkowski J, Elantkowska M, Dembczyński J. Semi-empirical calculations of oscillator strengths and hyperfine structure constants for Ti II. J Quant Spectrosc Radiat Transfer 2014;149:168.

[27] Bouazza S. Parametric study of hyperfine structure of $\mathrm{Zr}$ II even-parity levels. Can J Phys 2016;94:1310. 